\title{
Personality Disturbances as Emergent Phenomena Reflective of Underlying Neurobehavioral Systems: Beyond Dimensional Measurement, Phenotypic Trait Descriptors, and Factor Analysis
}

\author{
Mark F. Lenzenweger ${ }^{a-c}$ Richard A. Depue ${ }^{d}$ \\ ${ }^{a}$ Department of Psychology, The State University of New York at Binghamton, Binghamton, NY, USA; \\ ${ }^{b}$ Department of Psychiatry, Weill Cornell Medical College, New York, NY, USA; ' Personality Disorders Institute,

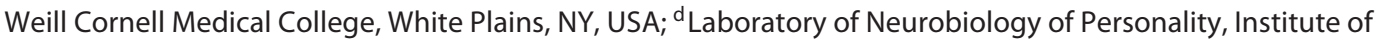 \\ Human Neuroscience, Department of Human Development, Cornell University, Ithaca, NY, USA
}

\section{Keywords \\ Neurobehavioral systems · Personality disorder · Emergent phenomena Personality neuroscience $\cdot$ DSM- 5 Alternative Model of Personality Disorders · Personality}

\begin{abstract}
Background and Overview: The conceptualization of personality pathology, or personality disturbance, is now at a substantive crossroads. Some researchers (and clinicians) prefer a focus on the domains of personality pathology that are well-described and captured in traditional categorical diagnostic approaches that, in some instances, abut normal personality constructs. Other workers argue to move the study of personality disorder (PD) closer to personality science seeking continuous connections between PD and established dimensions of healthy-range, normal personality. Most of the latter efforts revolve around correlational and factor analytic study of phenotypic expressions of PD features and normal personality dimensions. It is notable, how-
\end{abstract}

Correspondence: E-mail correspondence concerning this article may be directed to either Mark F. Lenzenweger, PhD, mlenzen@binghamton.edu or Richard A. Depue, PhD, rad5@cornell.edu. ever, that both visions of the PD/personality interface are essentially unlinked to an understanding of shared neurobiological underpinnings (i.e., neurotransmitter-influenced neurobehavioral systems) of both personality disturbance and normal personality ${ }^{1}$. Here, we present a nontechnical, conceptual overview of our approach to this problem, advancing a neurobehavioral approach that seeks to anchor both normal personality and personality disturbance within a matrix of brain-based neurobiological systems, incorporating genetic, epigenetic, and environmental inputs. In this brief paper, we seek only to provide a necessarily cursory introduction to how we conceptualize this area and illustrate, in broad outline, our effort to characterize both personality and personality disturbance anchored in neurobehavioral systems. Our approach, which we began developing in the middle 1990s, can be juxtaposed with the more recently proposed DSM-5 Alternative Model of Personality Disorders as well as the well-established five-factor approach to PD.

(c) 2020 S. Karger AG, Basel

In our modeling, we focus specifically on neurotransmitter systems and related neurobehavioral processes. We do not focus on functional neuroanatomy findings from neuroimaging studies of personality disordered participants, nor do we base our modeling on neuroimaging correlates of personality dimensions (e.g., five-factor approach).

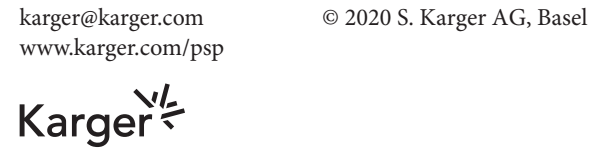




\section{Domains of Inquiry Converge: Brief Review of the Challenges to Integration}

Over the past 35 years, the field has seen efforts to understand clinical psychopathology constructs known as personality disorders (PDs) with personality science constructs that characterize models of normal-range personality. Prior to about 1985, these domains of constructs were quite separate in terms of both theory and empirical research [1]. Normal personality psychologists focused on dimensional trait conceptualizations, inventory development, and factor analytic studies of inventory data derived from, typically, university populations. Simultaneously, psychiatry and clinical psychology focused on the description and articulation of domains of PD that were long observed in the consulting room and conceived of categorically, but were less systematically delineated or conceptualized beyond atheoretical collections of signs and symptoms (e.g., antisocial personality, borderline pathology, narcissistic disorders, schizoid personality, and so on).

Two major foci of nearly all efforts seeking to bridge these two domains have been, importantly, to determine (a) whether the nature and breadth of normal personality constructs adequately map those thought to define the PD conceptual space and vice versa and (b) how the domains are fundamentally related (e.g., merely associated vs. genuinely continuous). From the 1980s through the early 2000s, a large empirical literature appeared that focused on the correlations that exist among normal personality constructs and PD concepts. The typical form of such studies was that a normal personality inventory and a measure of PD were administered to the same participants and the resulting associations across the two measures were described. Nearly all theoretical discussion regarding personality and PD sprung from empirical patterns discerned in these correlation matrices. Additionally, inspired in part by such data, many scholarly discussions of personality and PD during this time circled about the theme of "categories" versus "dimensions" and debated the best approach to conceptualization and measurement of PDs. Much of this work laid the foundation for what would eventually come to be known as the DSM-5 Alternative Model of Personality Disorder (AMPD) [2], a hybrid categorical-dimensional model of PD that incorporated much of what is contained in the well-known five-factor approach to normal personality plus some additional constructs of a more pathological nature.

A conspicuous feature of much of the initial work linking normal personality and PD was its reliance on corre- lational results showing associations between phenotypic measures of PD features and personality dimensions. The normal personality dimensions under consideration were most commonly the five personality factors/traits of neuroticism, extraversion, openness to experience, conscientiousness, and agreeableness. Such correlations amongst the PD and personality measures were argued, by some, as evidence reflective of continuity between personality and PD (e.g., elevated borderline PD features were often associated with low levels of agreeableness or related facet). Such correlations derived from manifest indicators, however, could not definitively confirm continuity in latent or underlying structure.

Another feature of this corpus pointed to the insufficiency of extant normal personality constructs for explaining the wide range and highly dysfunctional aspects of personality pathology (e.g., paranoia, self-mutilation, severe narcissism, profound amounts of aggression and meanness, near total absence of fear, micro-psychotic episodes, extreme masochism). Many experienced psychopathologists saw the major extant normal personality dimensions as simply too coarse and lacking in both nuance and configural relations to validly capture the PD domain, facet measures of personality dimensions notwithstanding. Efforts to link personality and PD in a deeper manner have been relatively sparse. Early on, Gray [3] sought to develop a model linking underlying neurophysiological systems to normal personality. An alternative approach to linking PD to normal personality systems, building upon the insights of Gray and others, was proposed by Cloninger et al. [4]. Their personality model consists of two broad classes of subdomains falling within temperament or character. PD is seen, in this model, as largely reflective of diminished self-directedness (a putative character feature) with modifying inputs from harmavoidance, novel-seeking, and/or reward-dependence, i.e., temperament factors.

Each of these issues (construct continuity, insufficient mapping of pathology, coarseness of construct conceptualization) remains unresolved to one degree or another today; however, the proposed DSM-5 AMPD has begun to advance the discussion considerably. For example, through the AMPD, there has been some degree of convergence regarding the likely domains of normal personality related to personality pathology, and a common language has been proposed. That the AMPD has not been uniformly accepted is attested to by its inclusion as an "emerging model" in the DSM-5 (Section III), whereas the official diagnostic criteria for PDs in DSM-5 remained unchanged from the prior system (DSM-IV-TR). A critical 
aspect that remains strikingly minimally developed in this area of inquiry, for both normal personality and $\mathrm{PD}$, is the underlying neurobiological influences at play in both normalcy and pathology in personality functioning. In this paper, we describe our approach to understanding personality and personality dysfunction from the standpoint of neurobehavioral systems and position it for comparison with the AMPD (see other papers in this special issue).

We present our model as an overview summary of our prior extended explications of our model of personality disturbance, personality, and neurobehavioral systems. We presented our initial neurobehavioral dimensional model of PD in 2001 [5], with subsequent revision in 2005 [6]. Our most recent model has been presented more recently in considerable detail elsewhere $[7,8]$. Here, we present our model succinctly in broad descriptive terms for consideration juxtaposed with the AMPD which is described extensively in the other papers in this special issue; the interested reader can consult our prior presentations for extensive technical neurobiological detail [5-8]. Our presentation focuses on (a) personality disturbance as emergent phenomena, which implicitly embraces a dimensional perspective, and (b) our specific neurobehavioral perspective that underpins both personality and personality disturbance.

\section{Personality Disturbance as Emergent Phenomena Arising from Underlying Dimensionally Structured Systems}

Since 2001, we have proposed that personality pathology be regarded as an emergent phenomenon [5]. Briefly, the concept of emergence and resultant emergent properties, when they arise, speak to the development or appearance of some novel condition or phenomenon that is coherent and integrated. An emergent phenomenon cannot be explained by reference to its constituent parts or contributing components. Stated differently, the emergent entity or phenomenon along with its properties cannot be predicted from the elements that make up the entity. In short, importantly, the emergent phenomenon is more than the simple sum of its parts. Emergence, as a descriptive organizing concept and process, plays a critical role in many areas of science. For example, emergent processes and properties figure centrally in fields as divergent as condensed matter and material physics to animal behavior to meteorology to contemporary cognitive neuroscience. There are many common examples of emergent phenomenon, such as bird flocking, the game of chess, hurricanes, and ant colonies. In psychological science, for example, one might think of the experience of consciousness or visual imagery as emergent properties of the brain (or, more specifically, neural circuits, neurobiological systems, and structures within the brain) [e.g., 9-11]. Furthermore, one could think of "rigidity" or "contour" as emergent properties of an object without reference to the physical components that make up an object. We [5$7,12]$ have argued that the concept of emergence is relevant to the psychopathology of the personality as well as most forms of disturbance represent complex configural outcomes of multiple interacting systems.

Specifically, we have argued that personality disorders as relatively discrete disease entities, such as conceived of within the traditional diagnostic nomenclatures, do not exist per se [6-8]. As an alternative, we proposed a perspective, where personality disturbance represents emergent phenotypes that arise from the interaction of extreme values on critical subsets of major personality systems, which are reflected in what are termed traits reflective of underlying neurobehavioral systems and structured dimensionally. That is, each individual's personality represents the interaction of all major underlying neurobehavioral systems. In this context, we note that the term emergent is used to describe personality pathology as configurations of disturbance that "emerge" or "arise" from the novel combination of constituent personality systems, but such configurations of disturbance cannot be reduced or parsed readily into those contributing neurobehavioral systems. Where individuals lie at the extreme (low or high) ends of interacting trait dimensions on subsets of the major neurobehavioral systems (which we identify phenotypically as traits), emotional behavior emerges that generates social and/or occupational impairment, rendering disturbance in the individual's life resulting from these personality systems.

This general model follows from our position that higher-order traits of personality reflect the activities of neurobehavioral systems [7, 12-17]. Though genetically speaking it may be possible to conceive of the neurobiological variables associated with neurobehavioral systems as subject to independent influences, it is impossible to imagine that these systems are independent at a functional level. Neurobehavioral systems, and the personality traits that reflect their influence, interact to produce complex behavior patterns - personality as a whole - in a multivariate, interactive fashion [see 7,12 , and below for more discussion of this issue]. Although this model is fully dimensional in nature, where personality disturbance lies at the extreme of normal, interacting personality di- 
Fig. 1. A modeling strategy for deriving neurobiological hypotheses about higherorder traits of personality and personality disturbance. Copyright ${ }^{(C)} 2014$ by R.A. Depue and M.F. Lenzenweger. Used with permission.

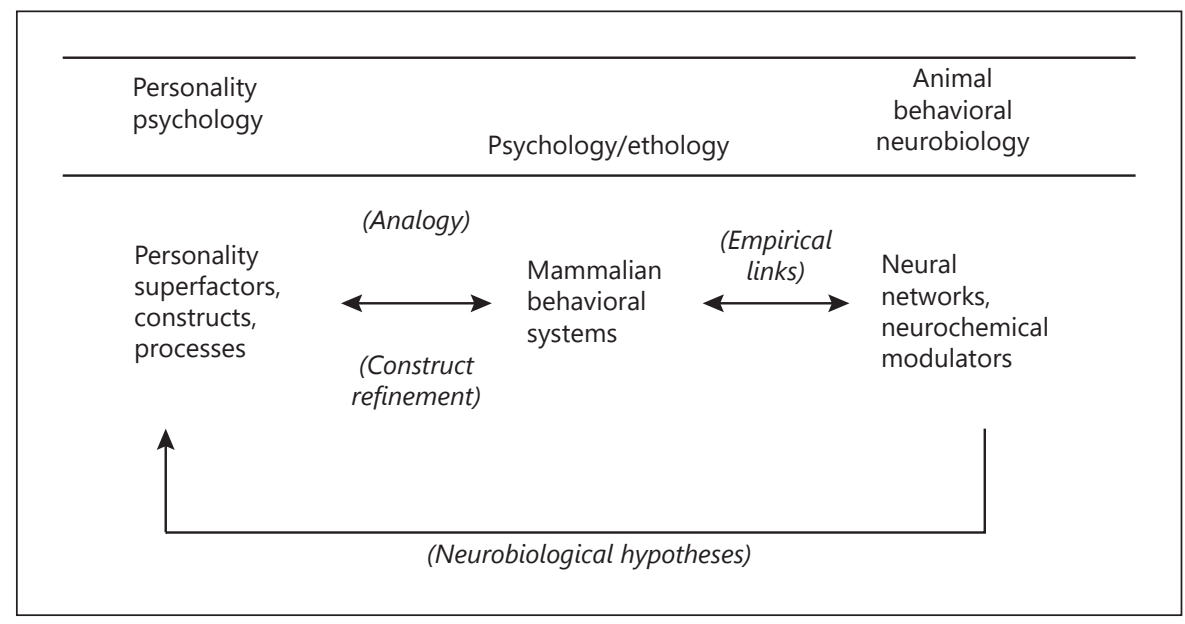

mensions, no assumption is made herein that phenotypic dimensions of personality are genetically continuous. The phenotypic continuity could well represent several underlying distinct genotypic distributions, as may be the case even within the normal range of variation of some personality traits [13].

\section{Conceptual Overview of a Neurobehavioral Model Underpinning Both Personality and Personality Disturbance}

Before reviewing our model, we believe it is important to address a prior model of PD that embraces neurobiological determinants. It is one that views PD largely in terms of subsyndromal or preclinical expressions of liabilities for major forms of psychopathology (i.e., psychotic illness, affective disorders, and so on). Siever and colleagues $[18,19]$ proposed that PDs represented subsyndromal expressions of major forms of psychopathology. Borderline PD, for example, is viewed as a variant of affective disorder. Borderline PD, in Siever et al.'s approach, is viewed essentially as a subsyndromal variant of affective illness marked by affective instability as a central criterion as well as high levels of impulse dyscontrol and aggression, thus seen largely as pathological dysfunction in serotonergic functioning. Importantly, as distinguished from our model, the Siever model fails to capture the interactive nature of multiple underlying neurobiological processes that set the stage for personality pathology.

In developing our neurobehavioral model of personality disturbance, we extended the strategy originally described in Depue and Collins [14] to personality distur- bance as outlined in Figure 1. Personality psychology was used to define the behavioral, emotional, and motivational characteristics of molar dimensions of normal-range human personality (e.g., extraversion). Next, we identified mammalian behavior patterns with corresponding characteristics, as described in the psychological and ethological literatures. Once an analogous motivation was identified, the animal neurobiological research was consulted, and that corpus provided empirical links to its neural organization and neurochemical modulation. These hypotheses were then extended to humans and were defined in a manner allowing for empirical tests. Once the major neurobehavioral systems were identified through this process, we sought to conceptualize wellknown patterns of personality disturbance within the multidimensional space defined by these systems [5-7].

Our multidimensional model of personality disturbance is illustrated in Figure 2. In the model, the axes are defined by neurobehavioral systems $[7,8,14-17,20]$ rather than by personality traits, because traits are only approximate, fallible (phenotypic) estimates of these systems. It is also essential to note that we do not seek to place the established categorical DSM-system PDs within this multidimensional construct space. In a prior effort, we [5] demonstrated that one could approximate the location of DSM PDs in such a multidimensional space. However, our current view [7-8] is that the model allows for a richer depiction of regions of personality dysfunction depicted in the mapped reaction surface in Figure 2. The regions of dysfunction accord well with variation on the underlying neurobehavioral systems, would reveal themselves in phenotypic disturbance, and do not require reliance on DSM PD constructs. 
Our model consists of multiple neurobehavioral systems: incentive motivation, anxiety/stress reactivity, affiliative reward, rejection sensitivity, neural (nonaffective) constraint, affective aggression, and fear. Furthermore, each of these neurobehavioral systems is modulated by one or more neurotransmitter systems of wide brain distribution [for reviews, see 7, 8, 12, 15, 20]. In Figure 2, two major neurobehavioral systems are modeled as a ratio of relative strength, because the opposing nature of their eliciting stimuli affects behavior in a reciprocal manner, such that the elicitation or expression of one system is influenced by the strength of the other: (1) a dopamine-facilitated system of behavioral approach based on positive incentive motivation, which is hypothesized to underlie extraversion and positive emotionality $[12,14,16]$; and (2) norepinephrine and corticotrophin-releasing hormone-modulated anxiety/stress reactivity $[12,15,21]$, thought to underlie neuroticism and negative emotionality $[6,7,12,22]$.

Similarly, a $m u$-opiate and oxytocin-modulated affiliative reward dimension $[17,20,23]$, representing the capacity to experience consummatory reward from social and soft-touch stimulation [20], and thought to underlie the trait of social closeness or agreeableness [17], is placed in ratio with rejection sensitivity. A ratio of these 2 systems is modeled, because they both largely influence the interpersonal domain but can have opposite effects on social bonding. Moreover, a dimension of rejection sensitivity likely provides a novel contribution to the traditional categories of dependent-, avoidant-, and borderline disturbance (all high on this dimension).

Finally, a neural constraint dimension is hypothesized to underlie the trait of constraint or conscientiousness. Through the interaction of serotonin, GABA, and norepinephrine, constraint provides inhibitory modulation of the threshold of stimulus elicitation of the other neurotransmitter systems $[12,15,24-27]$. Hence, in turn, the behavioral expressive features of the other neurobehavioral systems are also modulated by constraint. Due to the limitation of graphing more than 3 dimensions, it should be noted that the model includes one additional system/trait input that we suggest influences the manifestation of personality disturbance: the ratio of affective aggression:fear to account for a full range of antisocial behavior (particularly, high aggression:low fear). Thus, taken together, the model proposes that the interaction of multiple neurobehavioral-neurotransmitter systems underlying dimensional personality traits is necessary to account for the emergent phenotypes observed in personality disturbance.

Neurobehavioral Model of Personality Disturbances as Emergent Phenomena

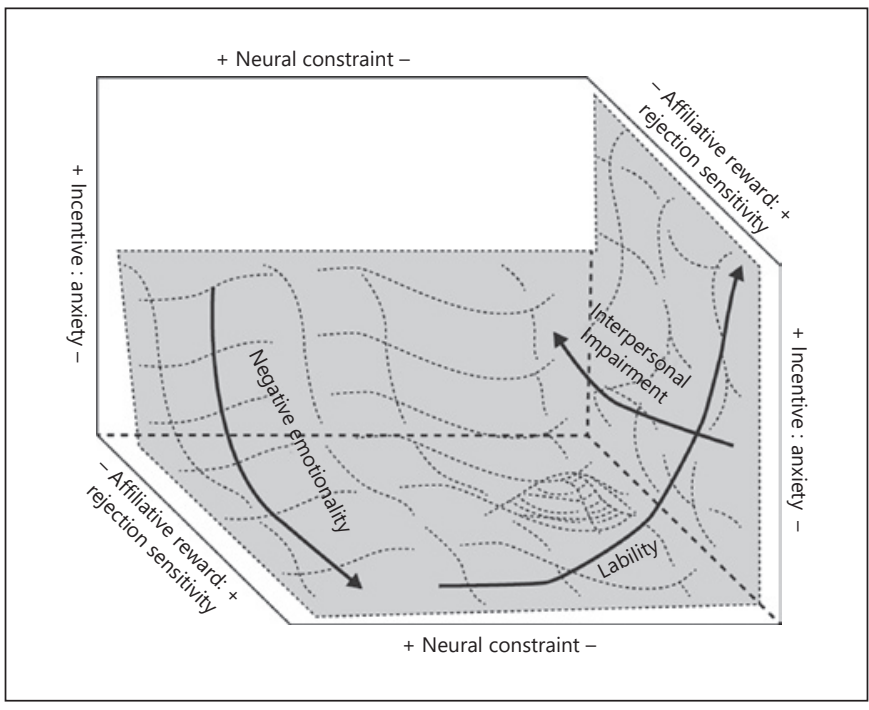

Fig. 2. A multidimensional neurobehavioral model of personality disturbance. The incentive, affiliative reward, anxiety, neural constraint, and rejection sensitivity neurobehavioral systems are depicted in terms of a reaction surface. Copyright ${ }^{\circ} 2014$ by R.A. Depue and M.F. Lenzenweger. Used with permission.

Three significant features of the model in Figure 2 are worth emphasizing. First, the phenotypic expression of personality disturbance, represented by the gray-shaded reaction surface in the figure (accounting for $\sim 10 \%$ of the population [28]), is continuous in nature, changing in character gradually but seamlessly across the surface in a manner that reflects the changing product of the multidimensional interactions. Because the functioning of the neurotransmitter systems underlying neurobehavioral systems is interactive in nature, estimating the product of these multidimensional interactions most effectively is an important task for future research. One framework for such estimation was recently proposed, and it serves as an initial step in the process [12].

Second, it may be that certain areas of the reaction surface (i.e., certain system/trait interactions) are associated with increased probability (risk) of certain features of personality disturbance, perhaps having given rise initially to clinical theories of PDs based on prototypic phenotypes. An example of such increased probability is represented in the right-back area of the figure as an elevation in the surface that is continuous rather than distinct in contour. As noted above, this representation, however, is not meant to imply that a distinct disorder exists in that particular zone of the surface.

Importantly, the extent and positioning of the gray reaction surface is weighted most heavily by increasing anx- 
iety/stress reactivity, decreasing neural constraint, and decreasing affiliative reward:increasing rejection sensitivity. This weighting is also reflected in the lines with directional arrows overlaying the gray surface in Figure 2, which illustrate increasing phenotypic negative emotionality, emotional lability, and interpersonal impairment. These three factors were found to be the most common characteristics of all PDs in a meta-analysis [29], and a study by Kendler et al. [30] showed independent genetic contributions to these factors, thereby empirically supporting their emphasis in the model.

Third, our modeling of personality disturbance accounts for the nature of dysfunction found to occur in most well-known forms of personality disturbance [2, Section I] irrespective of reliance on presumed DSM categorical structure. Importantly, in this context, we note that we have advocated for a view that both schizotypal and paranoid disturbances are less likely to be effectively modeled as variants of personality and its underlying neurobehavioral substrates. This position is informed by the substantial corpus of data that identifies these forms of psychopathology as intimately related to schizophrenia per se rather than personality. We have argued that these conditions more accurately represent alternative manifestations of the liability for schizophrenia [31, 32]. As such, we have not sought to model schizotypal and paranoid pathology within our neurobehavioral framework (in this context, we also note the AMPD posits a "psychoticism" personality factor, which we do not include in our framework as per our discussion of schizophreniarelated pathology).

There are other points of divergence in our model vis a vis the DSM-5 AMPD [2, Section III]. For example, we see the positive emotion/incentive reward system as playing a critical role in the emergence of normal personality as well as personality disturbance, particularly in relation to anxiety, whereas positive emotion does not play a central role in the DSM-5 AMPD. An expanded AMPD would need to incorporate explicitly positive emotion. We also note our explicit focus on rejection sensitivity as an important dimension/process within our proposed model, which is an important point of divergence from the AMPD. The AMPD describes "separation insecurity," but only as a sub-domain of negative emotion, which is not necessarily reflective solely of rejection sensitivity. In our modeling, we seek to avoid blended phenotypic constructs such as "impulsivity," which reflects at least five systems, such as positive incentive motivation, fear, affective and competitive aggression, and nonaffective constraint. Rather, we prefer a focus on the nature of the un- derlying neurobehavioral systems, which in interaction allow for the emergence of heterogenous, blended behavioral phenotypic outcomes.

Many additional implications of our model are discussed in detail elsewhere [7-8], but two seem important in the consideration of the development of personality disturbance. First, each of the neurotransmitters discussed above are subject to significant and long-lasting modification in functioning by early postnatal experience $[12,20]$. Exactly which types of early experience are most impactful on which neurotransmitters is currently an area of intense research [20]. Nevertheless, it is clear that genetically influenced effects on neurotransmitter functioning alter human perceptual, cognitive, motor, and affective responses to environmental contexts $[14,16,20$, 23]. Thus, in this framework, elevation and dynamic movement in the reaction surface of the model could be strongly influenced by current but historically meaningful environmental contexts. Therefore, it would seem that this area of research could profitably be integrated with clinical and research accounts of personality disturbance.

Second, sex differences in some forms of personality disturbance may imply differential risk (neurobiological and/or environmental) based on biological sex and/or psychological gender-related influences. The increased prevalence of males with antisocial behavior may reflect the higher mean of males in the population on the ratio of traits of aggression:fear and a lower mean on constraint $[26,27]$. Additionally, the increased prevalence of females with borderline- and dependent-like personality disturbance seen in some samples (though not all) may reflect a combination of (a) their higher mean on both trait anxiety; (b) their higher mean on social closeness, thereby increasing the need for social relationships whose loss is feared; (c) their higher mean on rejection sensitivity, thereby increasing their vacillation between dependence and rejection of others; (d) their lower mean on extraversion, particularly social dominance, hence enhancing the predominance of high levels of trait anxiety; and (e) their lower mean on the ratio of aggression:fear, hence decreasing the prevalence of antisocial behavior $[7,12,14,17$, 33].

We see neurobiologically informed study of the development of personality disturbance, particularly as related to the appearance of sex differences, as an area ripe for future research. For example, while one could pursue numerous vectors of research, issues of particular importance are (a) how subcortical neural systems of approach and avoidance develop much sooner than cortical neural systems of self-regulation; (b) how cortical neural devel- 
opment is compromised in many PDs; and (c) how environmental insults can affect cortical development [34]. Inclusion of evolutionary perspectives on a neurobiologically-based model of personality and personality disturbance development could also be considered [e.g., 35]. Finally, it would be useful to seek connections among the neurobehavioral systems indicators that we discuss here and clinically relevant measures of functional impairment.

We offer this neurobehavioral perspective on the emergence of personality and personality disturbance for consideration vis a vis other personality approaches, such as the five-factor approach and the AMPD. For example, while the five-factor conceptualizations (five-factor model, Big-5) of personality and PDs maintain their traction in some quarters, the AMPD has gained a substantial foothold in the scientific study of PD since its introduction in 2013 [2]. However, what remains striking is that both the five-factor approach as well as the AMPD approach remain largely disconnected from neurobiological modeling; rather, they are anchored in the correlational and factor analytic frameworks that seek to explore the interface of personality and personality disturbance through patterns of association of phenotypic indicators. We offer our model as a heuristic and substantive explanation of the underlying nature of both personality and personality disturbance. We believe it suggests an integrated basis for understanding both personality and personality disturbance while simultaneously suggesting im- portant vectors for empirical research across levels of analysis, ranging from the genetic and epigenetic [see 7 for extensive discussion of genetic and epigenetic considerations], through neurobiology, to neurocognitive processes [36] and phenotypic individual differences, all the while allowing for interpersonal [37] and environmental inputs.

\section{Statement of Ethics}

Ethics approval was not required for this report as it is a statement of theory.

\section{Conflict of Interest Statement}

The authors have no conflicts of interest to declare.

\section{Funding Sources}

This theory development work was supported in part by $\mathrm{Na}$ tional Institute of Mental Health Research Grants MH-45448 to M.F. Lenzenweger and MH-55347 to R.A. Depue.

\section{Author Contributions}

Mark F. Lenzenweger: theory development, PDs, personality; Richard A. Depue: theory development, PDs, personality.

\section{References}

1 Widiger TA, Frances A. The DSM-III personality disorders. Perspectives from psychology. Arch Gen Psychiatry. 1985 Jun;42(6):615-23.

2 American Psychiatric Association. Diagnostic and statistical manual of mental disorders (DSM-5). 5th ed. Arlington (VA): American Psychiatric Publishing; 2013.

3 Gray JA. The psychology of fear and stress. Problems in the behavioural sciences. Volume 5. 2nd ed. Cambridge, England: Cambridge University Press; 1987.

4 Cloninger CR, Svrakic DM, Przybeck TR. A psychobiological model of temperament and character. Arch Gen Psychiatry. 1993 Dec; 50(12):975-90.

5 Depue RA, Lenzenweger MF. A neurobehavioral dimensional model of personality disorders. In: Livesley WJ, editor. The Handbook of Personality Disorders. New York: Guilford 2001. pp. 136-76.

6 Depue RA, Lenzenweger MF. A neurobehavioral model of personality disturbance. In: Lenzenweger MF, Clarkin JF, editors. Major theories of personality disorder. 2nd ed. New York: Guilford; 2005. pp. 391-453.

7 Lenzenweger MF, Depue RA. Toward a developmental psychopathology of personality disturbance: A neurobehavioral dimensional model incorporating genetic, environmental, and epigenetic factors. In: Cicchetti D, editor. Maladaptation and Psychopathology. Developmental Psychopathology. Volume 3. 3rd ed. New York: Wiley; 2016. pp. 1079-110.

8 Depue R, Fu Y. Neurogenetic and experiential processes underlying major personality traits: implications for modelling personality disorders. Int Rev Psychiatry. 2011 Jun;23: 258-81.

9 Rumelhart DE. The emergence of cognitive phenomena from sub-symbolic processes. In: Proceedings of the Sixth Annual Conference of the Cognitive Science Society. Hillsdale, NJ: Lawrence Erlbaum; 1984. pp. 59-62.

10 Kosslyn SM, Pomerantz JR. Imagery, propositions, and the form of internal representations. Cognit Psychol. 1977;9(1):52-76.
11 McClelland JL, Botvinick MM, Noelle DC, Plaut DC, Rogers TT, Seidenberg MS, et al. Letting structure emerge: connectionist and dynamical systems approaches to cognition. Trends Cogn Sci. 2010 Aug;14(8):348-56.

12 Moore SR, Depue RA. Neurobehavioral foundation of environmental reactivity. Psychol Bull. 2016 Feb;142(2):107-64.

13 Depue RA. Interpersonal behavior and the structure of personality: Neurobehavioral foundation of agentic extraversion and affiliation. In: Canli T, editor. Biology of Personality and Individual Differences. New York: Guilford Press; 2006. pp. 60-92.

14 Depue RA, Collins PF. Neurobiology of the structure of personality: dopamine, facilitation of incentive motivation, and extraversion. Behav Brain Sci. 1999 Jun;22(3):491-517.

15 Depue RA, Fu Y. The neurobiology and neurochemistry of temperament in adults. In: Zentner M, Shiner RL, editors. The Handbook of Temperament. New York: Guilford Press; 2012. pp. 368-99.
Neurobehavioral Model of Personality Disturbances as Emergent Phenomena
Psychopathology 2020;53:213-220 DOI: 10.1159/000509624 
16 Depue RA, Fu Y. On the nature of extraversion: variation in conditioned contextual activation of dopamine-facilitated affective, cognitive, and motor processes. Front Hum Neurosci. 2013 Jun;7:288.

17 Depue RA, Morrone-Strupinsky JV. A neurobehavioral model of affiliative bonding: implications for conceptualizing a human trait of affiliation. Behav Brain Sci. 2005 Jun;28(3): 313-50.

18 Siever LJ, Davis KL. A psychobiological perspective on the personality disorders. Am J Psychiatry. 1991 Dec;148(12):1647-58.

19 Siever LJ, Weinstein LN. The neurobiology of personality disorders: implications for psychoanalysis. J Am Psychoanal Assoc. 2009 Apr;57(2):361-98.

$20 \mathrm{Fu}$ Y, Depue RA. A novel neurobehavioral framework of the effects of positive early postnatal experience on incentive and consummatory reward sensitivity. Neurosci Biobehav Rev. 2019 Dec;107:615-40.

21 White TL, Depue RA. Differential association of traits of fear and anxiety with norepinephrine- and dark-induced pupil reactivity. J Pers Soc Psychol. 1999 Oct;77(4):863-77.

22 Canli T. Toward a neurogenetic theory of neuroticism. Ann N Y Acad Sci. 2008;1129(1): 153-74.

23 Fu Y, Selcuk E, Moore SR, Depue RA. Touchinduced face conditioning is mediated by genetic variation in opioid but not oxytocin receptors. Sci Rep. 2018;8:9004.
24 Carver CS, Miller CJ. Relations of serotonin function to personality: current views and a key methodological issue. Psychiatry Res. 2006 Sep;144(1):1-15.

25 Depue RA, Spoont MR. Conceptualizing a serotonin trait. A behavioral dimension of constraint. Ann N Y Acad Sci. 1986;487:47-62.

26 Spoont MR. Modulatory role of serotonin in neural information processing: implications for human psychopathology. Psychol Bull. 1992 Sep;112(2):330-50.

27 Zald DH, Depue RA. Serotonergic functioning correlates with positive and negative affect in psychiatrically healthy males. Pers Individ Dif. 2001 Jan;30(1):71-86.

28 Lenzenweger MF, Lane MC, Loranger AW, Kessler RC. DSM-IV personality disorders in the National Comorbidity Survey Replication. Biol Psychiatry. 2007 Sep;62(6):553-64.

29 Saulsman LM, Page AC. The five-factor model and personality disorder empirical literature: A meta-analytic review. Clin Psychol Rev. 2004 Jan;23(8):1055-85.

30 Kendler KS, Aggen SH, Czajkowski N, Røysamb E, Tambs K, Torgersen S, et al. The structure of genetic and environmental risk factors for DSM-IV personality disorders: a multivariate twin study. Arch Gen Psychiatry. 2008 Dec;65(12):1438-46.

31 Lenzenweger MF. Schizotypy and schizotypic psychopathology: Mapping an alternative expression of schizophrenia liability. In: Lenzenweger MF, Dworkin RH, editors. Origins and development of schizophrenia: Advances in experimental psychopathology. Washington (DC): American Psychological Association; 1998. pp. 93-121.
32 Lenzenweger MF. Schizotypy and schizophrenia: The view from experimental psychopathology. New York: Guilford Press; 2010. ISBN 978-1-60623-865-3.

33 Tellegen A, Waller NG. Exploring personality through test construction: development of the Multidimensional Personality Questionnaire. In: Boyle GJ, Matthews G, Saklofske DH, editors. The SAGE handbook of personality theory and assessment, Vol. 2. Personality measurement and testing. London, England: Sage Publications; 2008. pp. 261-292.

34 Beauchaine TP. A developmental psychopathology perspective on the emergence of antisocial and borderline personality pathologies across the lifespan: commentary on personality pathology in youth. In: Lejuez CW, Gratz $\mathrm{KL}$, editors. The Cambridge handbook of personality disorders. New York, NY: Cambridge University Press; 2020. pp. 94-98.

35 Panksepp J. Affective neuroscience: The foundations of human and animal emotions. Oxford, England: Oxford University Press; 1998.

36 Lenzenweger MF, Clarkin JF, Fertuck EA, Kernberg OF. Executive neurocognitive functioning and neurobehavioral systems indicators in borderline personality disorder: a preliminary study. J Pers Disord. 2004 Oct; 18(5): 421-38.

37 Savage M, Lenzenweger MF. The impact of social exclusion on "Reading the Mind in the Eyes" performance in relation to borderline personality disorder features. J Pers Disord. 2018 Feb;32(1):109-30. 